\title{
EFECTO DEL TOLTRAZURIL AL 2.5\% DURANTE EL PERIODO PREPATENTE DE LA SARCOCISTIOSIS INTESTINAL CANINA
}

\author{
EFFECT O F TO LTRAZURIL $2.5 \%$ DURING THE PREPATENT PERIOD O F CANINE \\ SARCOCYSTIOSIS
Julio Vilca M. ${ }^{1}$, Feliciana Vilca S. ${ }^{2}$, Amanda Chávez V. ${ }^{3}$, Martín Urviola $S .{ }^{2}$ y Víctor Leyva V. ${ }^{4}$

\section{Resumen}

El objetivo del estudio fue determinar el efecto del toltrazuril al 2.5\% en dosis única para el tratamiento de la sarcocistiosis intestinal de perros infectados experimentalmente con Sarcocystis lamacanis. Se utilizaron 24 cachorros cruzados de ambos sexos, de 2.5 meses de edad, previamente desparasitados y alimentados con dieta libre de carne cruda. Los perros fueron infectados con cerca de 95,000 quistes de $S$. lamacanis, presentes en $100 \mathrm{~g}$ de músculo cardiaco de alpaca. Se les distribuyó en un grupo control no tratado de 4 cachorros y cuatro grupos de 5 animales c/u tratados con toltrazuril al $2.5 \%$ en dosis única de 10 ó $20 \mathrm{mg} / \mathrm{kg}$ de peso vivo, administrado oralmente al $5^{\circ}$ ó $7^{\circ}$ día post-infección. Las muestras fecales fueron recolectadas diariamente, a partir del $10^{\circ}$ día post-infección y por 11 días. Se analizó las heces por el método de concentración por flotación con solución Sheather para determinar la presencia de esporoquistes, y las muestras positivas se analizaron con el método de Stoll modificado para determinar el número de esporoquistes por gramo de heces. El toltrazuril no logró controlar la infección por $S$. lamacanis en caninos en ninguno de sus tratamientos.

Palabras clave: toltrazuril, sarcocistiosis, control, efectividad, coccidiosis

\section{AbSTRACT}

The aim of the study was to determine the effect of toltrazuril (2.5\%) in a single dose for the treatment of canine sarcocystiosis. Twenty-four crossbred puppies, both sexes, 2.5 months of age, were used. All puppies were dewormed prior to the study and fed throughout the study with a diet free of raw meat. Animals were experimentally infected with nearly 95,000 cysts of $S$. lamacanis, contained in $100 \mathrm{~g}$ of alpaca heart, and distributed in a control (untreated) group of 4 puppies and 4 groups of 5 puppies each which were treated with toltrazuril (2.5\%) with a single dose of 10 or $20 \mathrm{mg} / \mathrm{kg}$ body weight on day 5 or 7 post-infection. Fecal samples were daily collected during 11 days and starting on day

\footnotetext{
${ }^{1}$ Práctica privada

${ }^{2}$ Facultad de Medicina Veterinaria y Zootecnia, UNA, Puno

${ }^{3}$ Laboratorio de Microbiología y Parasitología Veterinaria, FMV-UNMSM

E-mail: a-chavez-g@hotmail.com

${ }^{4}$ Laboratorio de Reproducción Animal, FMV-UNMSM
} 
10 post-infection. Feces was analyzed by the flotation method with the Sheather solution to determine the presence of sporocysts of Sarcocystis sp. and positive samples were analyzed by the modified Stoll method to determine the number of sporocysts per gram of feces. The toltrazuril did not control the infection of S. lamacanis in none of the treated groups.

Key words: toltrazuril, sarcocystosis, control, effectiveness, coccidiosis

\section{INTRODUCCIÓN}

La sarcocistiosis en los camélidos sudamericanos (CSA) es una de las enfermedades parasitarias que más afecta la eficiencia productiva y la productividad de alpacas y llamas, al formar quistes macroscópicos y microscópicos en los tejidos musculares. Esta enfermedad es ocasionada por el Sarcocystis aucheniae y $S$. lamacanis, respectivamente, los cuales son protozoos del grupo de Apicomplexos que poseen un ciclo de vida indirecto. Los hospederos definitivos son los caninos y los CSA son los hospederos intermediarios. Se estima pérdidas anuales cercanas a los 300 mil dólares solamente por el decomiso de carcasas afectadas por esta parasitosis (Leguía y Casas, 1999).

La elevada prevalencia de esta parasitosis, que en muchos casos alcanza hasta el $100 \%$ en animales mayores de 2 años de edad (Mostajo, 1983), afecta a cerca de 500 mil familias campesinas que dependen de la crianza de CSA, por lo que resulta de suma importancia realizar algún tipo de control que pueda ser aceptado por el poblador andino de bajos niveles socio-económicos y culturales. Actualmente, su control está orientado primordialmente a prevenir la enfermedad a través de la interrupción del ciclo de vida, tratando de evitar que los perros tengan acceso directo a las carnes infectadas con quistes de Sarcocystis sp. (Leguía y Casas, 1999). Lamentablemente, las limitadas campañas de educación sanitaria y la poca información sobre el problema han tenido poco impacto en la población afectada.

Estudios realizados en la Universidad Nacional del Altiplano de Puno, utilizando combinaciones de drogas como sulfadoxina y pirimetamina, así como primaquina, obtuvieron un $100 \%$ de eficacia en el control de la sarcocistiosis intestinal en caninos, luego de más de 7 días de tratamiento (Saravia, 2003; Quispe, 2004); sin embargo, se tiene la desventaja de su elevado costo y de requerir de aplicaciones por periodos prolongados. Otras drogas anticoccidiales presentes en el mercado local como el toltrazuril demostraron tener un efecto aceptable en todas las fases endógenas, incluyendo la esquizogonia y la gametogonia de otra apicomplexa como la Cystoisospora, además de ofrecer una alta eficacia con una sola aplicación (Daugschies et al., 2000). Estos resultados motivaron la realización del presente trabajo, a fin de evaluar esta droga en el perro como una nueva alternativa a la solución del problema de la sarcocistiosis en CSA. Por lo tanto, el objetivo del estudio fue determinar el efecto del toltrazuril al $2.5 \%$ en dosis única de 10 y 20 $\mathrm{mg} / \mathrm{kg}$ de peso vivo, aplicadas a los 5 y 7 días post infección para el tratamiento de la sarcocistiosis intestinal de perros infectados experimentalmente con $S$. lamacanis.

\section{Materiales y Métodos}

\section{Ubicación del estudio}

El estudio se realizó durante los meses de setiembre a diciembre del 2005 en la Estación Experimental del Centro de Investigaciones IVITA-Maranganí, a una altitud de 3,709 msnm, en la provincia de Canchis, región Cusco, de la Facultad de Medicina Veterinaria, Universidad Nacional Mayor de San Marcos. 
Cuadro 1. Programa de tratamiento con toltrazuril al $2.5 \%$ en caninos infectados experimentalmente con microquistes de Sarcocystis lamacanis

\begin{tabular}{lccc}
\hline Grupo & $\begin{array}{c}\text { Animales } \\
(\mathrm{n})\end{array}$ & $\begin{array}{c}\text { Dosis } \\
(\mathrm{mg} / \mathrm{kg} \mathrm{pv})\end{array}$ & $\begin{array}{c}\text { Día de administración } \\
\text { (post-infección) }\end{array}$ \\
\hline A & 5 & 10 & 5 \\
B & 5 & 10 & 7 \\
C & 5 & 20 & 5 \\
D & 5 & 20 & 7 \\
E (control) & 4 & - & - \\
\hline
\end{tabular}

\section{Manejo de los canes}

Se utilizaron 24 cachorros cruzados de ambos sexos, de aproximadamente 2.5 meses de edad. Los animales fueron desparasitados y permanecieron en observación por 14 días antes de iniciarse el experimento. Recibieron alimento balanceado comercial, libre de carne cruda, y se les suministró agua ad libitum.

Los canes fueron alojados en casetas individuales (caniles) de madera, con dimensiones de 1.0 x 0.80 x $0.80 \mathrm{~m}$. Las casetas se lavaron y desinfectaron con lejía antes de introducir a los cachorros y luego en forma semanal. El piso de las jaulas fue cubierto con papel periódico que se cambió con frecuencia.

Se utilizó tejido cardiaco de alpacas positivas a S. lamacanis, obtenido del camal de Sicuani. Los cachorros fueron infectados, vía oral, con 100 g de músculo cardiaco, conteniendo aproximadamente 940 microquistes por gramo de corazón.

\section{Procedimiento experimental}

A cada grupo experimental se le aplicó toltrazuril al $2.5 \%$ por vía oral de acuerdo al esquema de dosificación correspondiente (Cuadro 1). La droga se administró en ayunas con la ayuda de una jeringa hipodérmica sin aguja.
Muestras fecales fueron recolectadas diariamente por las mañanas a partir del día 10 post-infección y por 11 días. El análisis coprológico se realizó mediante el método de concentración por flotación con solución azucarada o de Sheather con el objetivo de determinar la presencia de esporoquistes en heces. Las muestras positivas fueron cuantificadas mediante el método de Stoll modificado para determinar el número de esporoquistes por gramo de heces.

\section{Análisis estadístico}

La variable número de esporoquistes en heces por grupo de tratamiento fue analizada bajo un arreglo factorial de 2 × 2 mediante el diseño de Bloque Completo al Azar.

Las diferencias entre grupos de tratamiento (número de esporoquistes) se determinaron mediante análisis de varianza. Se utilizó la prueba de Tukey (Daniel, 1996) para determinar diferencias entre promedios.

\section{Resultados y Discusión}

El toltrazuril al $2.5 \%$, tanto en la dosis de 10 como de $20 \mathrm{mg} / \mathrm{kg}$ de peso vivo, aplicadas al $5^{\circ}$ ó $7^{\circ}$ día de la infección no fue significativamente efectivo para inhibir la formación de ooquistes y la consecuente eliminación de esporoquistes de $S$. lamacanis durante los 11 días de observación (Cuadro 2). 
Cuadro 2. Efecto de la aplicación del toltrazuril al 2.5\% durante el periodo patente, expresado como número de esporoquistes por gramo de heces (epg) en el control del Sarcocystis lamacanis

\begin{tabular}{|c|c|c|c|c|c|}
\hline \multirow{2}{*}{$\begin{array}{l}\text { Periodo } \\
\text { patente } \\
\text { (días) }\end{array}$} & \multicolumn{5}{|c|}{ Tratamientos } \\
\hline & $\begin{array}{c}10 \mathrm{mg} / \mathrm{kg} \\
\text { Día } 5\end{array}$ & $\begin{array}{c}10 \mathrm{mg} / \mathrm{kg} \\
\text { Día } 7\end{array}$ & $\begin{array}{c}20 \mathrm{mg} / \mathrm{kg} \\
\text { Día } 5\end{array}$ & $\begin{array}{c}20 \mathrm{mg} / \mathrm{kg} \\
\text { Día } 7\end{array}$ & Control \\
\hline 1 & 920 & 520 & 480 & 800 & 750 \\
\hline 2 & 840 & 400 & 480 & 600 & 750 \\
\hline 3 & 800 & 360 & 520 & 840 & 1,400 \\
\hline 4 & 1,120 & 520 & 1,120 & 1,160 & 1,300 \\
\hline 5 & 1,080 & 480 & 1,240 & 840 & 1,600 \\
\hline 6 & 920 & 360 & 840 & 1,320 & 1,650 \\
\hline 7 & 1,720 & 600 & 840 & 1,240 & 1,250 \\
\hline 8 & 1,040 & 520 & 1,520 & 1,160 & 1,500 \\
\hline 9 & 1,360 & 560 & 1,200 & 1,520 & 1,200 \\
\hline 10 & 1,440 & 480 & 1,760 & 2,040 & 1,400 \\
\hline 11 & 1,280 & 480 & 1,720 & 2,050 & 1,400 \\
\hline Prom \pm d.e. & $1,138 \pm 285$ & $480 \pm 78$ & $1,065 \pm 475$ & $1,236 \pm 486$ & $1,291 \pm 300$ \\
\hline
\end{tabular}

El grupo que recibió toltrazuril en dosis de $10 \mathrm{mg} / \mathrm{kg}$ en el día 7 post-infección tuvo una reducción significativa $(\mathrm{p}>0.05)$ en la carga de esporoquistes con respecto a los demás grupos tratados. Existe la posibilidad de haber obtenido una mejor respuesta si se hubiera efectuado aplicaciones adicionales, tal como lo hizo Rommel et al. (1987) en el tratamiento de toxoplasmosis en gatos quien obtuvo mejores resultados con una medicación diaria de toltrazuril durante la fase prepatente en comparación con la dosis única que no pudo inhibir la formación de ooquistes de Toxoplasma gondii.

Hay evidencias que el toltrazuril tiene un efecto coccidicida sobre el desarrollo del Sarcocystis a nivel intracelular (Sumano y Ocampo, 1997), destruyendo las formas sexuales o gametocitos, por lo que puede ser usado como agente terapéutico. Así mismo, Daugschies et al. (2000) lo usaron favora- blemente en dosis única contra cystoisosporosis en perros infectados experimentalmente con ooquistes.

Los resultados encontrados demostrarían que con una sola dosis del toltrazuril no evitarían la formación de ooquistes y probablemente los días de administración ( $5^{\circ}$ y $7^{\circ}$ post-infección) no fueron adecuados, ya que este fármaco actúa en la fase sexual o gametogonia del Sarcocystis, dañando los micro y macrogametocitos; y esta fase estaría ocurriendo entre los primeros días de infección de los cachorros (Cordero del Campillo et al., 1999), es decir, en las primeras 18 horas de la infección.

La infección con S. lamacanis en caninos ha podido ser controlada experimentalmente con otras drogas. Saravia (2003) utilizó con éxito la combinación sulfadoxinapirimetamina al $9^{\circ}$ día del tratamiento, en tan- 
to que Quispe (2004) lo consiguió con primaquina al $7^{\circ}$ día del tratamiento. Paralelamente, Yujra (2004) trabajó con la combinación sulfadoxina-pirimetamina al $6^{\circ}$ día del tratamiento de una infección con macroquistes. Sin embargo, y pese al éxito de estos tratamientos a nivel experimental, no es posible su aplicación práctica debido a su elevado costo y a la necesidad de tratamientos diarios prolongados. Se requiere continuar con los trabajos de evaluación del toltrazuril, así como otras drogas en diferentes posologías, para lograr un adecuado control práctico y económico de la sarcocistiosis en CSA.

\section{Conclusiones}

El toltrazuril al $2.5 \%$ en dosis de 10 ó 20 $\mathrm{mg} / \mathrm{kg}$ de peso vivo, aplicado al $5^{\circ}$ ó $7^{\circ}$ día post-infección no fue efectivo para el control de la sarcocistiosis intestinal en perros infectados experimentalmente con microquistes.

\section{Literatura Citada}

1. Cordero de Campillo, M.; F. Rojo; M. Fernández; M. Sánchez; S. Rodríguez; I. López. 1999. Parasitología veterinaria. p 319-362. Mc Graw-Hill Interamericana. Madrid.

2. Daniel, D. 1996. Bioestadística base para el análisis de las ciencias de la salud. $5^{\text {ta }}$ ed. Ed. Uteha. p 138-170. México DF.

3. Daugschies, A; H. Mundt; V. Letkova. 2000. Toltrazuril treatment of cystoisosporosis in dogs under experimental and field conditions. Parasitol. Res. 86: 797-799.
4. Leguía, G; E. Casas. 1999. Enfermedades parasitarias y atlas parasitológico de camélidos sudamericanos. p 23-29. Ed. De Mar. Lima, Perú.

5. Mostajo, W. 1983. Sarcocistiosis en alpacas beneficiadas en el camal municipal de Santa Rosa. Tesis de Médico Veterinario y Zootecnista. Facultad de Medicina Veterinaria y Zootecnia, Univ. Nacional del Altiplano. Puno. 68 p.

6. Quispe, A. 2004. Efectividad de la primaquina, sulfaquinoxalina y primaquina-sulfaquinoxalina en el tratamiento de la sarcocistiosis en perros infectados experimentalmente con quistes microscópicos y macroscópicos. Tesis de Médico Veterinario y Zootecnista. Facultad de Medicina Veterinaria y Zootecnia, Univ. Nacional del Altiplano. Puno. 75 p.

7. Rommel, M; T. Schnieder; H. Krause; J. Westerhott. 1987. Ensayo de inhibición de la ooquisto y quistogénesis de Toxoplasma gondii en gatos, mediante la medicación del alimento con toltrazuril. Noticias Med. Vet. 2: 141-152.

8. Saravia, Z. 2003. Anticoccidiales en el tratamiento de la sarcocistiosis en perros infectados con microquistes de alpaca. Tesis de Médico Veterinario y Zootecnista. Facultad de Medicina Veterinaria y Zootecnia, Univ. Nacional del Altiplano. Puno.

9. Sumano M.; L. Ocampo. 1997. Farmacología veterinaria. $2^{a}$ ed. p 284290. Ed. Interamericana. México.

10. Yujra, F. 2004. Anticoccidiales en el tratamiento de sarcocistiosis en perros infectados con macroquistes de alpaca. Tesis de Médico Veterinario y Zootecnista. Facultad de Medicina Veterinaria y Zootecnia, Univ. Nacional del Altiplano. Puno. 